\title{
The effect of free glycerol intake on cerebral glycerol concentration
}

\author{
Adamkov $\mathbf{J}^{1}$, Poczos $\mathrm{P}^{1,2}$, Kanta $\mathrm{M}^{1}$, Habalova $\mathrm{J}^{1}$, Bartos $\mathrm{M}^{1}$, Travnicek $\mathrm{P}^{1}$, Mejzlik $\mathrm{J}^{3}$, Cesak $\mathrm{T}^{1}$ \\ Department of Neurosurgery, University Hospital Hradec Králové and Charles University, Faculty of Medicine \\ in Hradec Kralove, Czech Republic. jaroslav.adamkov@lfhk.cuni.cz
}

\begin{abstract}
OBJECTIVE: Cerebral microdialysis (CMD) is a method used to measure the concentration of metabolites and glycerol in the interstitium of the brain. The aim of this study was to investigate the effect of parenterally applied medication and nutrition containing external free glycerol (EFG) on cerebral values of glycerol in patients monitored and treated for non-traumatic subarachnoid hemorrhage (SAH).

METHODS: In 13 patients, the values of CG concentrations were measured using CMD. The amounts of parenterally applied EFG (in hourly intervals) were calculated from patient records. All data were gathered retrospectively. To analyze the association between the parameters of interest and their relationship,

Spearman's correlation and p-values were calculated.

RESULTS: There was no evident relationship between the CG and EFG concentrations when the dataset was analyzed as a whole $(r=-0.146)$. However, when the analysis was applied to single patients, a varying degree of correlations was discovered in 7 patients $(r=0.431-0.867)$.

CONCLUSION: The possible effect of externally administered glycerol contained in pharmaceuticals and nutrition on its brain concentrations must be considered when interpreting data of CMD (Tab. 2, Fig. 4,

Ref. 16). Text in PDF www.elis.sk

KEY WORDS: glycerol, microdialysis, brain, subarachnoid hemorrhage.
\end{abstract}

\section{Introduction}

Despite modern diagnostic and therapeutic methods, subarachnoid hemorrhage continues to carry a high level of morbidity and mortality, which is due to ruptured cerebral aneurysm. A chain of events which results in alteration of metabolism of brain cells likely contributes to the poor outcomes $(1,2)$. In some patients, the products of hemoglobin degradation and lower availability of vasodilation substances lead to vasoconstriction of blood vessels of various diameters, and as a consequence, the cerebral perfusion is reduced (3). Over time, an energetic disbalance in membrane transport systems leads to an influx of calcium ions into the cells (4). The elevated intracellular calcium concentration causes the activation of phospholipases resulting in degradation of membrane phospholipids. The end result of this chain of events is an increase in local glycerol concentration $(4,5)$.

The concentration of glycerol in areas of hypoxia/ischemia is 15 times higher when compared to physiological values. The

${ }^{1}$ Department of Neurosurgery, University Hospital Hradec Kralove and Charles University, Faculty of Medicine in Hradec Kralove, Czech Republic, ${ }^{2}$ Department of Anatomy, Faculty of Medicine in Hradec Kralove, Charles University, Czech Republic, and ${ }^{3}$ Department of Otorhinolaryngology and Head and Neck Surgery, University Hospital Hradec Kralove and Charles University, Faculty of Medicine in Hradec Kralove, Czech Republic

Address for correspondence: J. Adamkov, MD, Department of Neurosurgery, Sokolska 581, CZ- 50005 Hradec Kralove, Czech Republic. Phone: +420.776384367 normalization of glycerol concentration is observed with a relatively large delay ( 96 hours after the SAH). This return to normal is mainly due to the diffusion and dilution of glycerol in the cerebral interstitium (6).

Cerebral microdialysis (CMD) is an invasive way of monitoring patients with brain damage (subarachnoid/intracerebral hemorrhage, traumatic brain injury). It enables to measure lactate, pyruvate, glutamate and glycerol concentrations in the extracellular compartment of the brain with the aim of detecting the ensuing metabolic changes. These changes may result in cerebral hypoxia, which is critical to the prognosis $(7,8)$.

Glycerol is one of the key metabolites analyzed in CMD. Its concentration may predict the severity of morphological damage to the cellular membranes of the brain (9). The specificity of glycerol in detecting structural lesions in the brain is relatively low (10). This is due to the fact that its cerebral concentration is affected by other non-cerebral factors. The concentration of glycerol in the brain may be influenced by its systemic (serum) concentration (10). The availability of serum glycerol increases after the mobilization of energetic sources due to lipolysis in adipose tissue (e.g. in a reaction to stress, such as subarachnoid hemorrhage). Parenteral administration of medications and nutrition containing free or bounded glycerol also increases the level of glycerol in plasma $(9,10)$ and very likely increases also the concentration of free glycerol in the cerebral compartment. Forssten et al. studied the influence of parenteral intake of substances such as propofol, parenteral nutrition, and insulin on cerebral interstitial glycerol concentrations (11). They did not find a significant correlation between intravenously 


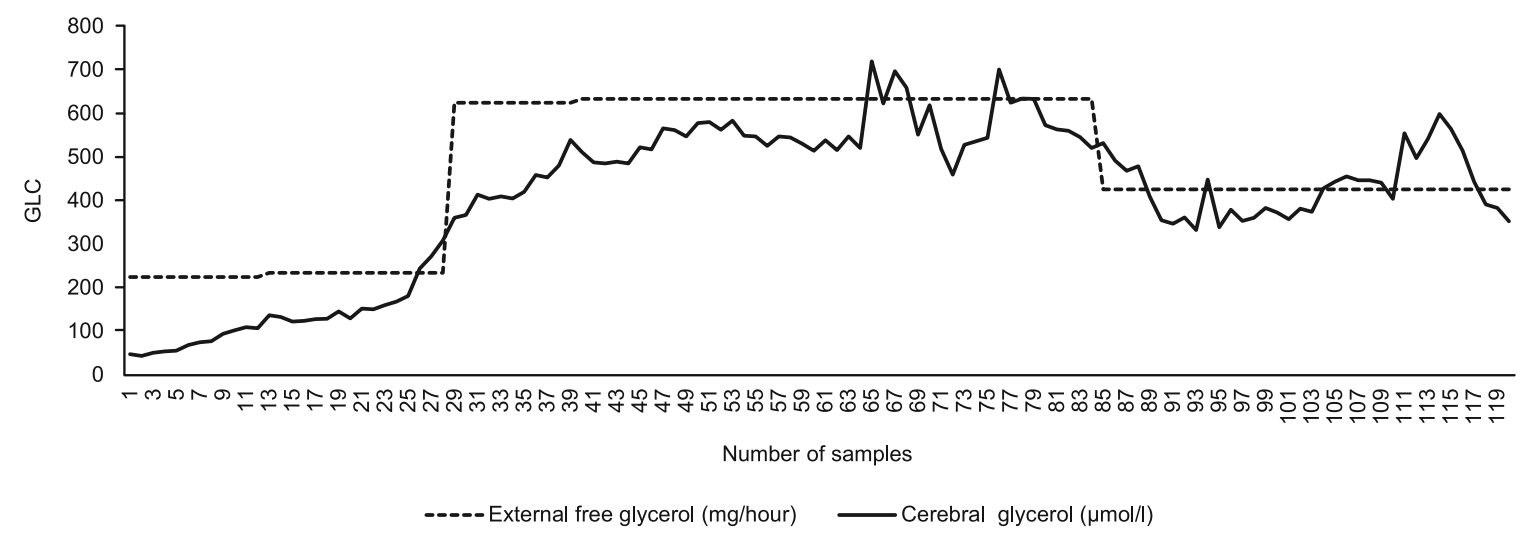

Fig. 1. An example of a direct and strong association between the tested parameters (patient No 1). GLC = glycerol.

administrated glycerol and CMD-glycerol levels (11). We believe this correlation may exist and thus we performed an analysis of our multimodally monitored patients with non-traumatic SAH.

\section{Materials and methods}

We carried out a retrospective analysis of records of all patients treated for non-traumatic SAH at the intensive care unit (ICU) of the Department of Neurosurgery at University Hospital Hradec Králové from January 2012 to December 2015. Thirteen patients, in whom early extubation following aneurysm treatment was unlikely, received microdialysis catheters as part of multimodal monitoring. The microdialysis catheter was implanted into the white matter of the non-dominant hemisphere when symmetrical blood distribution in the subarachnoid spaces was seen on baseline CT. When the blood occupied the basal cisterns and Sylvian fissure, the catheter was implanted into the frontal lobe, ipsilateral to the side of the bleeding. Multimodal monitoring started on day 1-3 following SAH. The speed of perfusion in the microdialysis catheter was set at $0.3 \mu 1 / \mathrm{min}$ using CMA70 and CMA71 catheters with membrane lengths of $10 \mathrm{~mm}$ and with pore sizes $20 \mathrm{kDA}$ and 100 $\mathrm{kDa}$, respectively. In order to measure the glycerol concentration, microdialysate samples were taken in hourly intervals and immediately processed with a bedside Iscus 600 analyser (uDialysis $\mathrm{AB}$, Stockholm, Sweden). Monitoring continued after obliteration of the source of hemorrhage which was done either surgically or endovascularly. The amount of parenterally administrated EFG

Tab. 1. Basic parameters of the patients' cohort.

\begin{tabular}{lc}
\hline Gender & Man=8, Woman $=5$ \\
Average age (years) & 48 \\
Average time of monitoring (hours) & 122 \\
Average free glycerol dose per 1 hour $(\mathrm{mg})$ & 318 \\
\hline
\end{tabular}

(mg/hour) was calculated from the intake of propofol and nutrition (Nutriflex Lipid Plus, Nutriflex Omega Plus, SmofKabiven, Vitalipid). The information on free glycerol concentration in parenteral medications was obtained from patients' daily documentation. Data on the concentration of free glycerol in propofol and nutrition was obtained from the manufacturers.

\section{Statistics}

Spearman's coefficient of rank correlation (r) was used to measure the relationship between EFG and cerebral glycerol (CG). The relationships for selected patients are presented on scatter graphs. For statistical analysis, we used NCSS 2019 Statistical Software, LLC. Kaysville, Utah, USA.

\section{Results}

Table 1 shows the characteristics of the group. There was no significant relationship between the CG and EFG concentrations when an average of the entire sample was analyzed $(r=-0.146)$. When individual patients were analyzed, the correlations ranged from negative values up to $r=0.867$. In 7 patients, the relationship between EFG and CG concentrations was strong $(r=0.431-0.868)$. The Spearman's correlation coefficients, $\mathrm{p}$ values and average hourly EFG in individual patients are presented in Table 2.

\section{Discussion}

The aim of this retrospective study was to assess the effect of parenterally administrated free glycerol contained in medications and nutrition on cerebral glycerol concentration. Previous publications have not shown a positive correlation between EFG and CG concentrations. Only an insignificant and clinically irrelevant

Tab. 2. Spearman's coefficients of rank correlation, $p$ and average amount of EFG in individual patients.

\begin{tabular}{|c|c|c|c|c|c|c|c|c|c|c|c|c|c|}
\hline Patient & 1 & 2 & 3 & 4 & 5 & 6 & 7 & 8 & 9 & 10 & 11 & 12 & 13 \\
\hline $\mathrm{R}$ & 0.867 & 0.742 & -0.095 & 0.809 & 0.243 & 0.077 & -0.254 & 0.620 & 0.667 & 0.182 & 0.431 & 0.831 & -0.039 \\
\hline $\mathrm{p}$ & $<0.001$ & $<0.001$ & 0.322 & $<0.001$ & 0.973 & 0.380 & 0.305 & $<0.001$ & $<0.001$ & 0.088 & $<0.001$ & $<0.001$ & 0.720 \\
\hline Glc & 476 & 343 & 316 & 225 & 114 & 217 & 301 & 579 & 232 & 374 & 414 & 222 & 331 \\
\hline
\end{tabular}

$\mathrm{R}$ - Spearman's coefficient of rank correlation, glc - average amount of EFG in mg/hour 


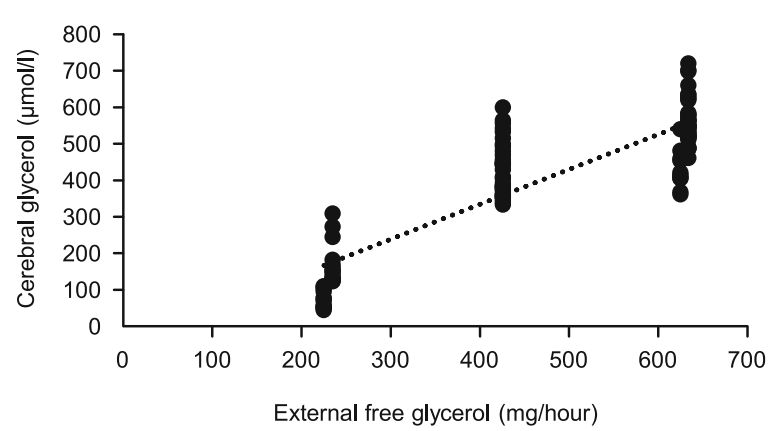

Fig. 2. Dependence (correlation) of CG on EFG (patient No 1).

correlation was found in a series of patients with traumatic brain injury (11). Here we show that when analysis of 13 individuals with non- traumatic SAH was performed, a correlation between EFG and CG concentrations could be seen in 7 patients. In 3 patients, the " $r$ " values were negative (indirect association). An example of direct and strong association between the tested parameters can be seen in patient No 1 (Figs 1 and 2). In comparison, patient No 7 is an example of an indirect association (Figs 3 and 4). The evidence here suggests that in some monitored patients, the concentration of CG may be influenced by EFG. If true, this raises the question of whether EFG disqualifies CG concentration analysis as a method to measure membrane damage. Further investigation is needed to determine this possibility. Figure 1 shows a marked similarity in the shape of both curves. We explain this as the effect of EFG on CG. To obtain a clearer explanation, it might be helpful to perform a parallel investigation of blood-brain barrier integrity using contrast enhanced magnetic resonance imaging. It is possible that in a subgroup of patients with $\mathrm{SAH}$, there is a disruption of the blood-brain barrier due to an energetic disbalance under hypoxic-ischemic conditions. This subsequently allows the escape of serum glycerol into the interstitium of the brain. In this case, the cerebral glycerol concentration would not correctly reflect the extent of membrane damage. In other words, the cerebral concentration of glycerol would be the sum of glycerol leakage through a damaged blood-brain barrier

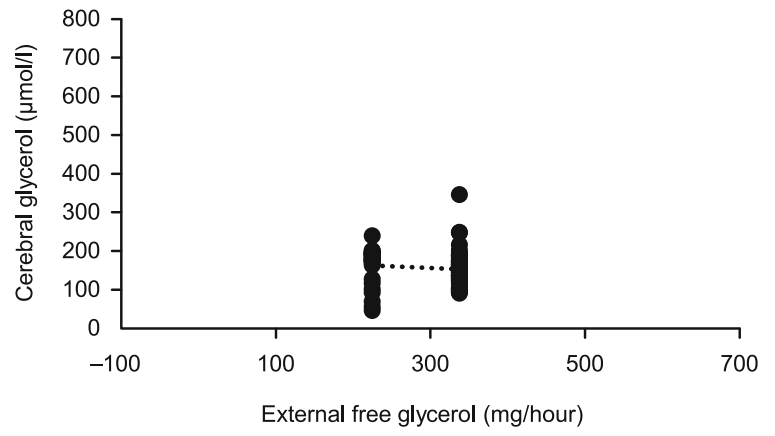

Fig. 4. Dependence (correlation) of CG on EFG (patient No 7).

(BBB) and cell membrane disintegration due to actual hypoxic condition. The degree of permeability of the BBB to glycerol may not be constant in time, and also there is a possibility of individual variability. A stronger correlation between CG and plasma concentrations would be expected when measuring the concentration of glycerol in juxtalesional locations such as contusions (catheter in proximity of the lesion, where there is a high possibility of BBB disruption). Nevertheless, a significant association between parenterally administrated glycerol and CG concentration in this kind of catheter placement in traumatic SAH has not been demonstrated (11).

In a study by Berger et al., cerebral microdialysis detected an accumulation of CG (up to $350 \%$ of the normal level) in patients with cerebral edema who received intravenous glycerol. The concentration of glycerol in brain tissue increased 20 minutes after its administration. A gradual return of CG to the baseline was seen afterwards. Cerebral hyperglycerolemia induced in this way lasted for 70 minutes. At the same time, the authors noticed only a slight increase in serum osmolarity (12). This knowledge leads us to the idea that an important and prompt passage of glycerol through the BBB could exist. Further, the rapid transport of glycerol across the BBB has been confirmed in patients with cerebral stroke in whom paralytic ileus was treated with glycerol inform of oral infusions. Maximum CG concentrations were reached 3-5 hours after glycerol administration (13).

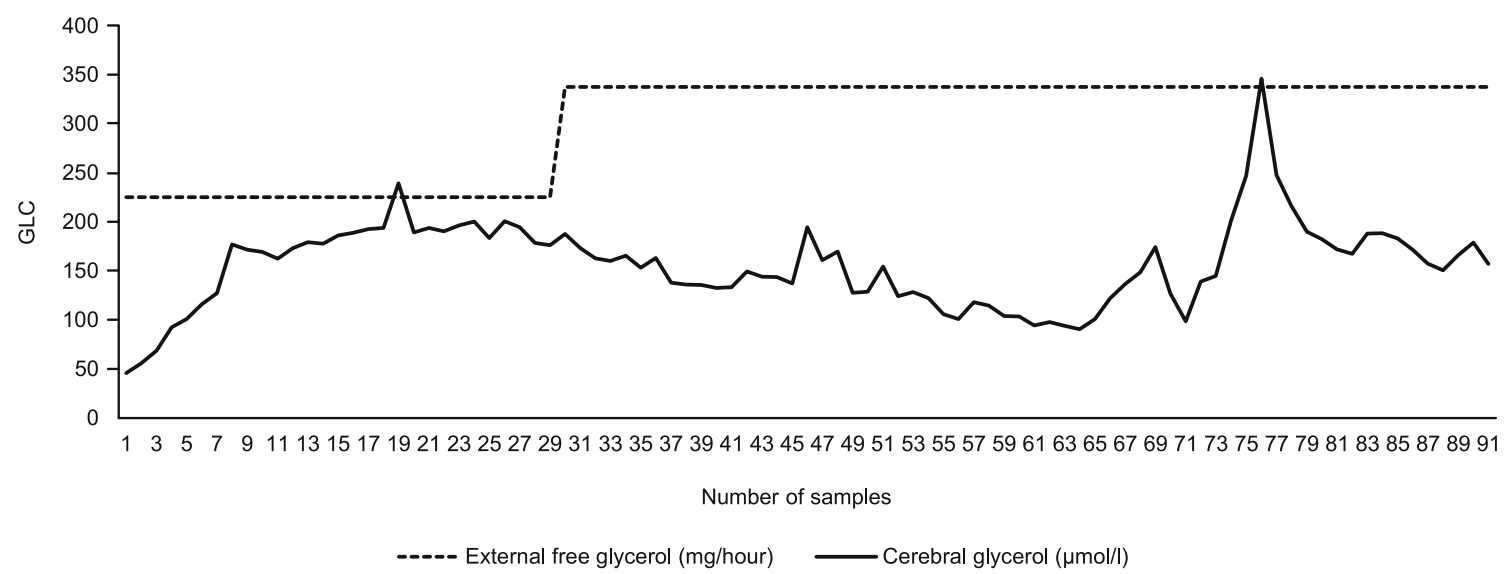

Fig. 3. An example of an indirect association between the tested parameters (patient No 7). GLC = glycerol. 
The conclusions of the aforementioned studies (11-13) suggest that the ability of EFG to affect CG could be dose-dependent. In the treatment of intracranial hypertension, the amount of one-time intravenously administered glycerol during 15 minutes was up to $25 \mathrm{~g}$. In contrast, in our study, the average dose of free glycerol from diet and parenteral intake was $318 \mathrm{mg}$ per hour. Repeated administration of glycerol contributes to its excessive accumulation in brain tissue. This can lead to a so-called rebound phenomenon when a paradoxical increase in intracranial pressure is seen after repetitive administration of an osmotically active drug (12). Due to this, glycerol is not currently used in clinical practice to treat intracranial hypertension. However, glycerol may have a positive effect on brain cells and their metabolism. In a hypoxic environment with glucose deficiency, glycerol presents an alternative source of energy (14). Glycerol favorably affects not only the regional brain perfusion and altered metabolism, but also the rheological properties of blood. This is due to the effect of leukocyte-endothelial interactions causing reduced ability of leukocytes to bind to blood elements and plasma (15).

Some forms of glycerol have the potential to disrupt bloodbrain barrier integrity (16). Alkylglycerols have an ability to transiently interfere with the integrity of the blood-brain barrier and increase its permeability. This property is utilized in oncology to increase the effect of certain cytostatics in the treatment of malignant brain tumors (16). It may be that acute stress reactions which contribute to a catecholamine response and result in hyperglycerolemia, when combined with administration of drugs and nutrition containing free glycerol, will similarly further disrupt the blood-brain barrier integrity or at least contribute to this process.

Further research on the effect of external glycerol intake on cerebral concentrations will require prospective studies with an emphasis on quantifying blood-brain barrier integrity. Although cerebral glycerol is not a parameter which could solely guide the clinician in treatment management, the knowledge of its pharmacokinetics and its association with blood-brain barrier integrity in SAH and TBI patients is useful.

The strength of this study lies in the relatively high number of measurements in each patient (on average $2 \times 122$ numerical values). Information regarding medications containing glycerol were accessed from patient charts from the ICU. Any changes in the speed of infusions, e.g. of propofol, were recorded in detail. Therefore, the total amount of EFG applied in each specific hour was calculated accurately. Our study has a limitation, namely in the small number of monitored patients and occasional delay in the analysis of CG samples due to late insertion of the microtube into the analyzer. Some values were acquired with a delay of up to 15 minutes.

\section{Conclusion}

There exists a correlation between external glycerol intake and cerebral glycerol concentration in some patients with nontraumatic SAH. It is likely to be due to the disruption in the bloodbrain barrier integrity with subsequent increased permeability to glycerol. The possible effect of externally administered glycerol contained in pharmaceuticals and nutrition on its brain concentrations must be considered when interpreting data of CMD.

\section{References}

1. Hütter BO, Kreitschmann-Andermahr I, Mayfrank L, Rohde V, Spetzger U, Gilsbach JM. Functional outcome after aneurysmal subarachnoid hemorrhage. Acta Neurochir Suppl 1999; 72: 157-174.

2. Hackett ML, Anderson CS. Health outcomes 1 year after subarachnoid hemorrhage: An international population-based study. The Australian Cooperative Research on Subarachnoid Hemorrhage Study Group. Neurology 2000; 55: 658-662.

3. Lin CL, Hsu YT, Lin TK, Morrow JD, Hsu JC, Hsu YH, Hsieh TC, Tsay PK, Yen HC. Increased levels of F2-isoprostanes following aneurysmal subarachnoid hemorrhage in humans. Free Radic Biol Med 2006; 40: 1466-1473.

4. Hubschmann OR, Nathanson DC. The role of calcium and cellular membrane dysfunction in experimental trauma and subarachnoid hemorrhage. J Neurosurg 1985; 62: 698-703.

5. de Lima Oliveira M, Kairalla AC, Fonoff ET, Martinez RC, Teixeira MJ, Bor-Seng-Shu E. Cerebral microdialysis in traumatic brain injury and subarachnoid hemorrhage: state of the art. Neurocrit Care 2014; 21: 152-162.

6. Hillered L, Valtysson J, Enblad P, Persson L. Interstitial glycerol as a marker for membrane phospholipid degradation in the acutely injured human brain. J Neurol Neurosurg Psychiatry 1998; 64 (4): 486-491.

7. Persson L, Valtysson J, Enblad P, Warme PE, Cesarini K, Lewen A, Hillered L. Neurochemical monitoring using intracerebral microdialysis in patients with subarachnoid hemorrhage. J Neurosurg 1996; 84 (4): 606-616.

8. Skjøth-Rasmussen J, Schulz M, Kristensen SR, Bjerre P. Delayed neurological deficits detected by an ischemic pattern in the extracellular cerebral metabolites in patients with aneurysmal subarachnoid hemorrhage. J Neurosurg 2004; 100 (1): 8-15.

9. Ungerstedt U, Rostami E. Microdialysis in neurointensive care. Curr Pharm Des 2004; 10 (18): 2145-2152.

10. Hutchinson PJ, Jalloh I, Helmy A, Carpenter KL, Rostami E, Bellander BM, Boutelle MG, Chen JW, Claassen J, Dahyot-Fizelier C et al. Consensus statement from the 2014 International Microdialysis Forum Intensive Care Med 2015; 41 (9): 1517-1528.

11. Forssten MP, Thelin EP, Nelson DW, Bellander BM. The Role of Glycerol-Containing Drugs in Cerebral Microdialysis: A Retrospective Study on the Effects of Intravenously Administered Glycerol. Neurocrit Care 2019; 30 (3): 590-600.

12. Berger C, Sakowitz OW, Kiening KL, Schwab S. Neurochemical monitoring of glycerol therapy in patients with ischemic brain edema. Stroke 2005; 36 (2): e4-e6.

13. Gliemroth J, Klaus S, Bahlmann L, Klöhn A, Duysen K, Reith A, Arnold H. Interstitial glycerol increase in microdialysis after glycerol enema. J Clin Neurosci 2004; 11 (1): 53-56.

14. Sloviter HA, Shimkin P, Suhara K. Glycerol as a substrate for brain metabolism. Nature 1966; 210 (5043): 1334-1336.

15. Meyer JS, Itoh Y, Okamoto S, Welch KM, Mathew NT, Ott EO, Sakaki S, Miyakawa Y, Chabi E, Ericsson AD. Circulatory and metabolic effects of glycerol infusion in patients with recent cerebral infarction. Circulation 1975; 51 (4): 701-712.

16. Erdlenbruch B, Kugler W, Schinkhof C, Neurath H, Eibl H, Lakomek M. Blood-brain barrier opening with alkylglycerols: Biodistribution of 1-O-pentylglycerol after intravenous and intracarotid administration in rats. J Drug Target 2005; 13 (3): 143-150.

Received September 1, 2020. Accepted November 27, 2020. 